\title{
\begin{tabular}{l|l} 
Mibraries & DSpace@MIT
\end{tabular}
}

\author{
MIT Open Access Articles
}

First Observation of Vector Boson Pairs in a Hadronic Final State at the Tevatron Collider

The MIT Faculty has made this article openly available. Please share how this access benefits you. Your story matters.

Citation: Aaltonen, T. et al. "First Observation of Vector Boson Pairs in a Hadronic Final State at the Tevatron Collider." Physical Review Letters 103.9 (2009): 091803. (C)2010 The American Physical Society.

As Published: http://dx.doi.org/10.1103/PhysRevLett.103.091803

Publisher: American Physical Society

Persistent URL: http://hdl.handle.net/1721.1/50758

Version: Final published version: final published article, as it appeared in a journal, conference proceedings, or other formally published context

Terms of Use: Article is made available in accordance with the publisher's policy and may be subject to US copyright law. Please refer to the publisher's site for terms of use. 


\section{First Observation of Vector Boson Pairs in a Hadronic Final State at the Tevatron Collider}

T. Aaltonen, ${ }^{24}$ J. Adelman, ${ }^{14}$ T. Akimoto, ${ }^{56}$ B. Álvarez González, ${ }^{12, u}$ S. Amerio, ${ }^{44 b, 44 a}$ D. Amidei, ${ }^{35}$ A. Anastassov, ${ }^{39}$ A. Annovi, ${ }^{20}$ J. Antos, ${ }^{15}$ G. Apollinari, ${ }^{18}$ A. Apresyan, ${ }^{49}$ T. Arisawa, ${ }^{58}$ A. Artikov, ${ }^{16}$ W. Ashmanskas, ${ }^{18}$ A. Attal, ${ }^{4}$ A. Aurisano,${ }^{54}$ F. Azfar, ${ }^{43}$ W. Badgett, ${ }^{18}$ A. Barbaro-Galtieri, ${ }^{29}$ V. E. Barnes, ${ }^{49}$ B. A. Barnett,${ }^{26}$ P. Barria,${ }^{47,47 a}$ V. Bartsch, ${ }^{31}$ G. Bauer, ${ }^{33}$ P.-H. Beauchemin,${ }^{34}$ F. Bedeschi, ${ }^{47 a}$ D. Beecher,${ }^{31}$ S. Behari, ${ }^{26}$ G. Bellettini, ${ }^{47 b, 47 a}$ J. Bellinger, ${ }^{60}$ D. Benjamin, ${ }^{17}$ A. Beretvas, ${ }^{18}$ J. Beringer ${ }^{29}$ A. Bhatti, ${ }^{51}$ M. Binkley, ${ }^{18}$ D. Bisello, ${ }^{44 b, 44 a}$ I. Bizjak, ${ }^{31, z}$ R. E. Blair, ${ }^{2}$ C. Blocker, ${ }^{7}$ B. Blumenfeld, ${ }^{26}$ A. Bocci,${ }^{17}$ A. Bodek,${ }^{50}$ V. Boisvert ${ }^{50}$ G. Bolla, ${ }^{49}$ D. Bortoletto, ${ }^{49}$ J. Boudreau ${ }^{48}$ A. Boveia, ${ }^{11}$ B. Brau, ${ }^{11, b}$ A. Bridgeman, ${ }^{25}$ L. Brigliadori, ${ }^{6 b, 6 a}$ C. Bromberg, ${ }^{36}$ E. Brubaker,,${ }^{14}$ J. Budagov,${ }^{16}$ H. S. Budd,${ }^{50}$ S. Budd, ${ }^{25}$ S. Burke, ${ }^{18}$ K. Burkett, ${ }^{18}$ G. Busetto, ${ }^{44 b, 44 a}$ P. Bussey, ${ }^{22}$ A. Buzatu, ${ }^{34}$ K. L. Byrum, ${ }^{2}$ S. Cabrera, ${ }^{17, w}$ C. Calancha, ${ }^{32}$ M. Campanelli, ${ }^{36}$ M. Campbell, ${ }^{35}$ F. Canelli, ${ }^{14,18}$ A. Canepa,${ }^{46}$ B. Carls,${ }^{25}$ D. Carlsmith,${ }^{60}$ R. Carosi, ${ }^{47 a}$ S. Carrillo, ${ }^{19,0}$ S. Carron, ${ }^{34}$ B. Casal, ${ }^{12}$ M. Casarsa, ${ }^{18}$ A. Castro,${ }^{6 b, 6 a}$ P. Catastini,${ }^{47 c, 47 a}$ D. Cauz, ${ }^{55 b, 55 a}$ V. Cavaliere, ${ }^{47 c, 47 a}$ M. Cavalli-Sforza, ${ }^{4}$ A. Cerri, ${ }^{29}$ L. Cerrito, ${ }^{31, q}$ S. H. Chang, ${ }^{62}$ Y. C. Chen, ${ }^{1}$ M. Chertok, ${ }^{8}$ G. Chiarelli, ${ }^{47 a}$ G. Chlachidze, ${ }^{18}$ F. Chlebana, ${ }^{18}$ K. Cho, ${ }^{62}$ D. Chokheli, ${ }^{16}$ J. P. Chou, ${ }^{23}$ G. Choudalakis, ${ }^{33}$ S. H. Chuang, ${ }^{53}$ K. Chung, ${ }^{18, p}$ W. H. Chung, ${ }^{60}$ Y. S. Chung, ${ }^{50}$ T. Chwalek, ${ }^{27}$ C. I. Ciobanu, ${ }^{45}$ M. A. Ciocci, ${ }^{47 c, 47 a}$ A. Clark, ${ }^{21}$ D. Clark, ${ }^{7}$ G. Compostella, ${ }^{44 a}$ M. E. Convery, ${ }^{18}$ J. Conway, ${ }^{8}$ M. Cordelli, ${ }^{20}$ G. Cortiana, ${ }^{44 b, 44 a}$ C. A. Cox,${ }^{8}$ D. J. Cox,${ }^{8}$ F. Crescioli, ${ }^{47 b, 47 a}$

C. Cuenca Almenar, ${ }^{8, w}$ J. Cuevas, ${ }^{12, u}$ R. Culbertson, ${ }^{18}$ J. C. Cully, ${ }^{35}$ D. Dagenhart, ${ }^{18}$ M. Datta, ${ }^{18}$ T. Davies, ${ }^{22}$ P. de Barbaro, ${ }^{50}$ S. De Cecco, ${ }^{52 a}$ A. Deisher ${ }^{29}$ G. De Lorenzo, ${ }^{4}$ M. Dell'Orso,${ }^{47 b, 47 a}$ C. Deluca, ${ }^{4}$ L. Demortier,${ }^{51}$ J. Deng, ${ }^{17}$ M. Deninno, ${ }^{6 a}$ P. F. Derwent, ${ }^{18}$ A. Di Canto, ${ }^{47 b, 47 a}$ G. P. di Giovanni, ${ }^{45}$ C. Dionisi, ${ }^{52 b, 52 a}$ B. Di Ruzza, ${ }^{55 b, 55 a}$

J. R. Dittmann, ${ }^{5}$ M. D'Onofrio, ${ }^{4}$ S. Donati, ${ }^{47 b, 47 a}$ P. Dong, ${ }^{8}$ J. Donini,${ }^{44 a}$ T. Dorigo, ${ }^{44 a}$ S. Dube, ${ }^{53}$ J. Efron,${ }^{40}$ A. Elagin, ${ }^{54}$ R. Erbacher, ${ }^{8}$ D. Errede, ${ }^{25}$ S. Errede,${ }^{25}$ R. Eusebi,${ }^{18}$ H. C. Fang, ${ }^{29}$ S. Farrington, ${ }^{43}$ W. T. Fedorko, ${ }^{14}$ R. G. Feild, ${ }^{61}$

M. Feindt, ${ }^{27}$ J. P. Fernandez, ${ }^{32}$ C. Ferrazza, ${ }^{47 d, 47 a}$ R. Field, ${ }^{19}$ G. Flanagan, ${ }^{49}$ R. Forrest, ${ }^{8}$ M. J. Frank, ${ }^{5}$ M. Franklin ${ }^{23}$ J. C. Freeman, ${ }^{18}$ I. Furic,${ }^{19}$ M. Gallinaro, ${ }^{52 a}$ J. Galyardt,${ }^{13}$ F. Garberson, ${ }^{11}$ J. E. Garcia, ${ }^{21}$ A. F. Garfinkel, ${ }^{49}$ P. Garosi, ${ }^{47 c, 47 a}$ K. Genser ${ }^{18}$ H. Gerberich, ${ }^{25}$ D. Gerdes,${ }^{35}$ A. Gessler, ${ }^{27}$ S. Giagu, ${ }^{52 b, 52 a}$ V. Giakoumopoulou, ${ }^{3}$ P. Giannetti, ${ }^{47 a}$ K. Gibson, ${ }^{48}$ J. L. Gimmell,${ }^{50}$ C. M. Ginsburg, ${ }^{18}$ N. Giokaris, ${ }^{3}$ M. Giordani, ${ }^{55 b, 55 a}$ P. Giromini, ${ }^{20}$ M. Giunta, ${ }^{47 a}$ G. Giurgiu, ${ }^{26}$ V. Glagolev, ${ }^{16}$ D. Glenzinski, ${ }^{18}$ M. Gold,${ }^{38}$ N. Goldschmidt,${ }^{19}$ A. Golossanov, ${ }^{18}$ G. Gomez, ${ }^{12}$ G. Gomez-Ceballos, ${ }^{33}$ M. Goncharov ${ }^{33}$ O. González, ${ }^{32}$ I. Gorelov, ${ }^{38}$ A. T. Goshaw, ${ }^{17}$ K. Goulianos, ${ }^{51}$ A. Gresele, ${ }^{44 b, 44 a}$ S. Grinstein, ${ }^{23}$ C. Grosso-Pilcher, ${ }^{14}$ R. C. Group, ${ }^{18}$ U. Grundler, ${ }^{25}$ J. Guimaraes da Costa, ${ }^{23}$ Z. Gunay-Unalan, ${ }^{36}$ C. Haber ${ }^{29}$ K. Hahn, ${ }^{33}$ S. R. Hahn, ${ }^{18}$ E. Halkiadakis,${ }^{53}$ B.-Y. Han, ${ }^{50}$ J. Y. Han,${ }^{50}$ F. Happacher,${ }^{20}$ K. Hara,${ }^{56}$ D. Hare,${ }^{53}$ M. Hare, ${ }^{57}$ S. Harper, ${ }^{43}$ R. F. Harr, ${ }^{59}$ R. M. Harris, ${ }^{18}$ M. Hartz,${ }^{48}$ K. Hatakeyama, ${ }^{51}$ C. Hays, ${ }^{43}$ M. Heck, ${ }^{27}$ A. Heijboer, ${ }^{46}$ B. Heinemann, ${ }^{29}$ J. Heinrich, ${ }^{46}$ C. Henderson, ${ }^{33}$ M. Herndon, ${ }^{60}$ J. Heuser, ${ }^{27}$ S. Hewamanage, ${ }^{5}$ D. Hidas, ${ }^{17}$ C. S. Hill,,${ }^{11, d}$ D. Hirschbuehl, ${ }^{27}$ A. Hocker ${ }^{18}$ S. Hou, ${ }^{1}$ M. Houlden, ${ }^{30}$ S.-C. Hsu, ${ }^{29}$ B. T. Huffman,${ }^{43}$ R. E. Hughes, ${ }^{40}$ U. Husemann, ${ }^{61}$ M. Hussein,${ }^{36}$ J. Huston, ${ }^{36}$ J. Incandela, ${ }^{11}$ G. Introzzi, ${ }^{47 a}$ M. Iori,${ }^{52 b, 52 a}$ A. Ivanov, ${ }^{8}$ E. James,${ }^{18}$ D. Jang, ${ }^{13}$ B. Jayatilaka, ${ }^{17}$ E. J. Jeon, ${ }^{62}$ M. K. Jha, ${ }^{6 a}$ S. Jindariani, ${ }^{18}$ W. Johnson, ${ }^{8}$ M. Jones, ${ }^{49}$ K. K. Joo, ${ }^{62}$ S. Y. Jun,${ }^{13}$ J. E. Jung, ${ }^{62}$ T. R. Junk, ${ }^{18}$ T. Kamon, ${ }^{54}$

D. Kar, ${ }^{19}$ P. E. Karchin,${ }^{59}$ Y. Kato, ${ }^{42, \mathrm{n}}$ R. Kephart, ${ }^{18}$ W. Ketchum, ${ }^{14}$ J. Keung,${ }^{46}$ V. Khotilovich,${ }^{54}$ B. Kilminster, ${ }^{18}$ D. H. Kim ${ }^{62}$ H. S. Kim, ${ }^{62}$ H. W. Kim, ${ }^{62}$ J. E. Kim, ${ }^{62}$ M. J. Kim,${ }^{20}$ S. B. Kim, ${ }^{62}$ S. H. Kim, ${ }^{56}$ Y. K. Kim, ${ }^{14}$ N. Kimura, ${ }^{56}$

L. Kirsch, ${ }^{7}$ S. Klimenko, ${ }^{19}$ B. Knuteson, ${ }^{33}$ B. R. Ko, ${ }^{17}$ K. Kondo,${ }^{58}$ D. J. Kong, ${ }^{62}$ J. Konigsberg, ${ }^{19}$ A. Korytov, ${ }^{19}$ A. V. Kotwal, ${ }^{17}$ M. Kreps,${ }^{27}$ J. Kroll,,${ }^{46}$ D. Krop ${ }^{14}$ N. Krumnack, ${ }^{5}$ M. Kruse, ${ }^{17}$ V. Krutelyov, ${ }^{11}$ T. Kubo, ${ }^{56}$ T. Kuhr, ${ }^{27}$ N. P. Kulkarni, ${ }^{59}$ M. Kurata, ${ }^{56}$ S. Kwang, ${ }^{14}$ A. T. Laasanen, ${ }^{49}$ S. Lami, ${ }^{47 a}$ S. Lammel, ${ }^{18}$ M. Lancaster,${ }^{31}$ R. L. Lander, ${ }^{8}$

K. Lannon, ${ }^{40, t}$ A. Lath,${ }^{53}$ G. Latino, ${ }^{47,47 a}$ I. Lazzizzera, ${ }^{44 b, 44 a}$ T. LeCompte, ${ }^{2}$ E. Lee, ${ }^{54}$ H. S. Lee, ${ }^{14}$ S. W. Lee, ${ }^{54, v}$ S. Leone, ${ }^{47 a}$ J. D. Lewis, ${ }^{18}$ C.-S. Lin, ${ }^{29}$ J. Linacre, ${ }^{43}$ M. Lindgren, ${ }^{18}$ E. Lipeles, ${ }^{46}$ A. Lister, ${ }^{8}$ D. O. Litvintsev, ${ }^{18}$ C. Liu, ${ }^{48}$ T. Liu, ${ }^{18}$ N. S. Lockyer, ${ }^{46}$ A. Loginov, ${ }^{61}$ M. Loreti, ${ }^{44 b, 44 a}$ L. Lovas, ${ }^{15}$ D. Lucchesi, ${ }^{44 b, 44 a}$ C. Luci, ${ }^{52 b, 52 a}$ J. Lueck, ${ }^{27}$ P. Lujan, ${ }^{29}$ P. Lukens, ${ }^{18}$ G. Lungu,${ }^{51}$ L. Lyons,${ }^{43}$ J. Lys,${ }^{29}$ R. Lysak,${ }^{15}$ D. MacQueen,${ }^{34}$ R. Madrak ${ }^{18}$ K. Maeshima, ${ }^{18}$

K. Makhoul, ${ }^{33}$ T. Maki, ${ }^{24}$ P. Maksimovic,${ }^{26}$ S. Malde, ${ }^{43}$ S. Malik, ${ }^{31}$ G. Manca, ${ }^{30, f}$ A. Manousakis-Katsikakis, ${ }^{3}$

F. Margaroli, ${ }^{49}$ C. Marino, ${ }^{27}$ C. P. Marino, ${ }^{25}$ A. Martin, ${ }^{61}$ V. Martin, ${ }^{22,1}$ M. Martínez,${ }^{4}$ R. Martínez-Ballarín, ${ }^{32}$

T. Maruyama, ${ }^{56}$ P. Mastrandrea, ${ }^{52 a}$ T. Masubuchi, ${ }^{56}$ M. Mathis, ${ }^{26}$ M. E. Mattson,${ }^{59}$ P. Mazzanti, ${ }^{6 a}$ K. S. McFarland, ${ }^{50}$ P. McIntyre, ${ }^{54}$ R. McNulty, ${ }^{30, k}$ A. Mehta, ${ }^{30}$ P. Mehtala, ${ }^{24}$ A. Menzione, ${ }^{47 a}$ P. Merkel,${ }^{49}$ C. Mesropian,${ }^{51}$ T. Miao, ${ }^{18}$

N. Miladinovic, ${ }^{7}$ R. Miller, ${ }^{36}$ C. Mills,${ }^{23}$ M. Milnik, ${ }^{27}$ A. Mitra, ${ }^{1}$ G. Mitselmakher, ${ }^{19}$ H. Miyake, ${ }^{56}$ N. Moggi, ${ }^{6 a}$

M. N. Mondragon, ${ }^{18, o}$ C. S. Moon, ${ }^{62}$ R. Moore,${ }^{18}$ M. J. Morello, ${ }^{47 a}$ J. Morlock,${ }^{27}$ P. Movilla Fernandez,${ }^{18}$ J. Mülmenstädt, ${ }^{29}$ A. Mukherjee, ${ }^{18}$ Th. Muller, ${ }^{27}$ R. Mumford, ${ }^{26}$ P. Murat, ${ }^{18}$ M. Mussini, ${ }^{6 b, 6 a}$ J. Nachtman, ${ }^{18, p}$ Y. Nagai,${ }^{56}$ 
A. Nagano, ${ }^{56}$ J. Naganoma, ${ }^{56}$ K. Nakamura,${ }^{56}$ I. Nakano, ${ }^{41}$ A. Napier,${ }^{57}$ V. Necula,${ }^{17}$ J. Nett,${ }^{60}$ C. Neu, ${ }^{46, x}$ M. S. Neubauer, ${ }^{25}$ S. Neubauer, ${ }^{27}$ J. Nielsen, ${ }^{29, h}$ L. Nodulman, ${ }^{2}$ M. Norman, ${ }^{10}$ O. Norniella, ${ }^{25}$ E. Nurse,${ }^{31}$ L. Oakes, ${ }^{43}$ S. H. Oh, ${ }^{17}$ Y.D. Oh,${ }^{62}$ I. Oksuzian, ${ }^{19}$ T. Okusawa, ${ }^{42}$ R. Orava, ${ }^{24}$ K. Osterberg, ${ }^{24}$ S. Pagan Griso, ${ }^{44 b, 44 a}$ E. Palencia, ${ }^{18}$ V. Papadimitriou, ${ }^{18}$ A. Papaikonomou, ${ }^{27}$ A. A. Paramonov, ${ }^{14}$ B. Parks, ${ }^{40}$ S. Pashapour, ${ }^{34}$ J. Patrick, ${ }^{18}$ G. Pauletta, ${ }^{55 b, 55 a}$ M. Paulini, ${ }^{13}$ C. Paus,${ }^{33}$ T. Peiffer,${ }^{27}$ D. E. Pellett, ${ }^{8}$ A. Penzo ${ }^{55 a}$ T. J. Phillips,${ }^{17}$ G. Piacentino, ${ }^{47 a}$ E. Pianori, ${ }^{46}$ L. Pinera, ${ }^{19}$ K. Pitts, ${ }^{25}$ C. Plager, ${ }^{9}$ L. Pondrom, ${ }^{60}$ O. Poukhov, ${ }^{16, a}$ N. Pounder, ${ }^{43}$ F. Prakoshyn, ${ }^{16}$ A. Pronko, ${ }^{18}$ J. Proudfoot, ${ }^{2}$ F. Ptohos, ${ }^{18, j}$ E. Pueschel, ${ }^{13}$ G. Punzi, ${ }^{47 b, 47 a}$ J. Pursley, ${ }^{60}$ J. Rademacker, ${ }^{43, d}$ A. Rahaman, ${ }^{48}$ V. Ramakrishnan, ${ }^{60}$ N. Ranjan, ${ }^{49}$ I. Redondo, ${ }^{32}$ P. Renton, ${ }^{43}$ M. Renz,${ }^{27}$ M. Rescigno, ${ }^{52 a}$ S. Richter, ${ }^{27}$ F. Rimondi, ${ }^{6 b, 6 a}$ L. Ristori, ${ }^{47 a}$ A. Robson, ${ }^{22}$ T. Rodrigo, ${ }^{12}$ T. Rodriguez,${ }^{46}$ E. Rogers,${ }^{25}$ S. Rolli,${ }^{57}$ R. Roser,${ }^{18}$ M. Rossi,${ }^{55}$ R. Rossin, ${ }^{11}$ P. Roy ${ }^{34}$ A. Ruiz, ${ }^{12}$ J. Russ, ${ }^{13}$ V. Rusu, ${ }^{18}$ B. Rutherford,${ }^{18}$ H. Saarikko, ${ }^{24}$ A. Safonov, ${ }^{54}$ W. K. Sakumoto, ${ }^{50}$ O. Saltó, ${ }^{4}$ L. Santi, ${ }^{55 b, 55 a}$ S. Sarkar, ${ }^{52 b, 52 a}$ L. Sartori,${ }^{47 a}$ K. Sato, ${ }^{18}$ A. Savoy-Navarro, ${ }^{45}$ P. Schlabach,${ }^{18}$ A. Schmidt, ${ }^{27}$ E. E. Schmidt, ${ }^{18}$ M. A. Schmidt, ${ }^{14}$ M. P. Schmidt, ${ }^{61, a}$ M. Schmitt, ${ }^{39}$ T. Schwarz,${ }^{8}$ L. Scodellaro, ${ }^{12}$ A. Scribano, ${ }^{47 c, 47 a}$ F. Scuri, ${ }^{47 a}$ A. Sedov, ${ }^{49}$ S. Seidel, ${ }^{38}$ Y. Seiya,${ }^{42}$ A. Semenov, ${ }^{16}$ L. Sexton-Kennedy, ${ }^{18}$ F. Sforza,${ }^{47 b, 47 a}$ A. Sfyrla, ${ }^{25}$ S. Z. Shalhout, ${ }^{59}$ T. Shears, ${ }^{30}$ P. F. Shepard, ${ }^{48}$ M. Shimojima ${ }^{56, s}$ S. Shiraishi, ${ }^{14}$ M. Shochet, ${ }^{14}$ Y. Shon ${ }^{60}$ I. Shreyber ${ }^{37}$ P. Sinervo, ${ }^{34}$ A. Sisakyan, ${ }^{16}$ A. J. Slaughter, ${ }^{18}$ J. Slaunwhite, ${ }^{40}$ K. Sliwa,${ }^{57}$ J. R. Smith, ${ }^{8}$ F. D. Snider, ${ }^{18}$ R. Snihur, ${ }^{34}$ A. Soha, ${ }^{8}$ S. Somalwar, ${ }^{53}$ V. Sorin, ${ }^{36}$

T. Spreitzer, ${ }^{34}$ P. Squillacioti, ${ }^{47 c, 47 a}$ M. Stanitzki, ${ }^{61}$ R. St. Denis, ${ }^{22}$ B. Stelzer, ${ }^{34}$ O. Stelzer-Chilton, ${ }^{34}$ D. Stentz, ${ }^{39}$ J. Strologas, ${ }^{38}$ G. L. Strycker, ${ }^{35}$ J. S. Suh, ${ }^{62}$ A. Sukhanov, ${ }^{19}$ I. Suslov, ${ }^{16}$ T. Suzuki, ${ }^{56}$ A. Taffard, ${ }^{25, g}$ R. Takashima, ${ }^{41}$ Y. Takeuchi ${ }^{56}$ R. Tanaka, ${ }^{41}$ M. Tecchio, ${ }^{35}$ P. K. Teng, ${ }^{1}$ K. Terashi, ${ }^{51}$ J. Thom, ${ }^{18, i}$ A. S. Thompson, ${ }^{22}$ G. A. Thompson, ${ }^{25}$ E. Thomson, ${ }^{46}$ P. Tipton, ${ }^{61}$ P. Ttito-Guzmán, ${ }^{32}$ S. Tkaczyk, ${ }^{18}$ D. Toback,${ }^{54}$ S. Tokar,${ }^{15} \mathrm{~K}$. Tollefson,${ }^{36}$ T. Tomura, ${ }^{56}$

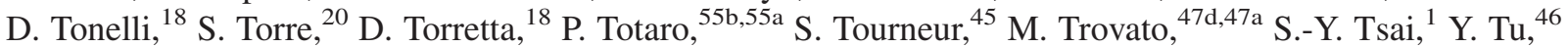

N. Turini, ${ }^{47 c, 47 a}$ F. Ukegawa, ${ }^{56}$ S. Vallecorsa, ${ }^{21}$ N. van Remortel,${ }^{24, c}$ A. Varganov, ${ }^{35}$ E. Vataga, ${ }^{47 d, 47 a}$ F. Vázquez, ${ }^{19,0}$ G. Velev ${ }^{18}$ C. Vellidis, ${ }^{3}$ M. Vidal,${ }^{32}$ R. Vidal,${ }^{18}$ I. Vila, ${ }^{12}$ R. Vilar, ${ }^{12}$ T. Vine,${ }^{31}$ M. Vogel, ${ }^{38}$ I. Volobouev, ${ }^{29, v}$ G. Volpi,${ }^{47 b, 47 a}$ P. Wagner, ${ }^{46}$ R. G. Wagner, ${ }^{2}$ R. L. Wagner, ${ }^{18}$ W. Wagner, ${ }^{27, y}$ J. Wagner-Kuhr, ${ }^{27}$ T. Wakisaka, ${ }^{42}$ R. Wallny, ${ }^{9}$ S. M. Wang, ${ }^{1}$ A. Warburton, ${ }^{34}$ D. Waters, ${ }^{31}$ M. Weinberger,${ }^{54}$ J. Weinelt, ${ }^{27}$ W. C. Wester III, ${ }^{18}$ B. Whitehouse,,${ }^{57}$ D. Whiteson, ${ }^{46, g}$ A. B. Wicklund, ${ }^{2}$ E. Wicklund, ${ }^{18}$ S. Wilbur, ${ }^{14}$ G. Williams, ${ }^{34}$ H. H. Williams,${ }^{46}$ P. Wilson, ${ }^{18}$ B. L. Winer,${ }^{40}$ P. Wittich, ${ }^{18, i}$ S. Wolbers, ${ }^{18} \mathrm{C}$. Wolfe, ${ }^{14} \mathrm{~T}$. Wright,${ }^{35} \mathrm{X}$. Wu, ${ }^{21} \mathrm{~F}$. Würthwein, ${ }^{10} \mathrm{~S}$. Xie, ${ }^{33} \mathrm{~A}$. Yagil, ${ }^{10} \mathrm{~K}$. Yamamoto, ${ }^{42} \mathrm{~J}$. Yamaoka, ${ }^{17}$ U. K. Yang, ${ }^{14, \mathrm{r}}$ Y. C. Yang, ${ }^{62}$ W. M. Yao, ${ }^{29}$ G. P. Yeh, ${ }^{18}$ K. Yi, ${ }^{18, p}$ J. Yoh, ${ }^{18}$ K. Yorita ${ }^{58}$ T. Yoshida, ${ }^{42, m}$ G. B. Yu, ${ }^{50}$ I. Yu, ${ }^{62}$ S. S. Yu, ${ }^{18}$ J. C. Yun, ${ }^{18}$ L. Zanello, ${ }^{52 b, 52 a}$ A. Zanetti, ${ }^{55 a}$ X. Zhang, ${ }^{25}$ Y. Zheng,,${ }^{9, e}$ and S. Zucchelli ${ }^{6 b, 6 a}$

\section{(CDF Collaboration)}

${ }^{1}$ Institute of Physics, Academia Sinica, Taipei, Taiwan 11529, Republic of China

${ }^{2}$ Argonne National Laboratory, Argonne, Illinois 60439

${ }^{3}$ University of Athens, 15771 Athens, Greece

${ }^{4}$ Institut de Fisica d'Altes Energies, Universitat Autonoma de Barcelona, E-08193, Bellaterra (Barcelona), Spain ${ }^{5}$ Baylor University, Waco, Texas 76798, USA

${ }^{6 a}$ Istituto Nazionale di Fisica Nucleare Bologna, I-40127 Bologna, Italy

${ }^{6 \mathrm{~b}}$ University of Bologna, I-40127 Bologna, Italy

${ }^{7}$ Brandeis University, Waltham, Massachusetts 02254, USA

${ }^{8}$ University of California, Davis, Davis, California 95616, USA

${ }^{9}$ University of California, Los Angeles, Los Angeles, California 90024, USA

${ }^{10}$ University of California, San Diego, La Jolla, California 92093, USA

${ }^{11}$ University of California, Santa Barbara, Santa Barbara, California 93106, USA

${ }^{12}$ Instituto de Fisica de Cantabria, CSIC-University of Cantabria, 39005 Santander, Spain

${ }^{13}$ Carnegie Mellon University, Pittsburgh, Pennsylvania 15213, USA

${ }^{14}$ Enrico Fermi Institute, University of Chicago, Chicago, Illinois 60637, USA

${ }^{15}$ Comenius University, 84248 Bratislava, Slovakia; Institute of Experimental Physics, 04001 Kosice, Slovakia

${ }^{16}$ Joint Institute for Nuclear Research, RU-141980 Dubna, Russia

${ }^{17}$ Duke University, Durham, North Carolina 27708

${ }^{18}$ Fermi National Accelerator Laboratory, Batavia, Illinois 60510, USA

${ }^{19}$ University of Florida, Gainesville, Florida 32611, USA

${ }^{20}$ Laboratori Nazionali di Frascati, Istituto Nazionale di Fisica Nucleare, I-00044 Frascati, Italy

${ }^{21}$ University of Geneva, CH-1211 Geneva 4, Switzerland

${ }^{22}$ Glasgow University, Glasgow G12 8QQ, United Kingdom

${ }^{23}$ Harvard University, Cambridge, Massachusetts 02138, USA 
${ }^{24}$ Division of High Energy Physics, Department of Physics, University of Helsinki and Helsinki Institute of Physics, FIN-00014, Helsinki, Finland

${ }^{25}$ University of Illinois, Urbana, Illinois 61801, USA

${ }^{26}$ The Johns Hopkins University, Baltimore, Maryland 21218, USA

${ }^{27}$ Institut für Experimentelle Kernphysik, Universität Karlsruhe, 76128 Karlsruhe, Germany

${ }^{28}$ Center for High Energy Physics: Kyungpook National University, Daegu 702-701, Korea; Seoul National University, Seoul 151-742, Korea; Sungkyunkwan University, Suwon 440-746, Korea;

Korea Institute of Science and Technology Information, Daejeon, 305-806, Korea;

Chonnam National University, Gwangju,500-757, Korea;

Chonbuk National University, Jeonju 561-756, Korea

${ }^{29}$ Ernest Orlando Lawrence Berkeley National Laboratory, Berkeley, California 94720, USA

${ }^{30}$ University of Liverpool, Liverpool L69 7ZE, United Kingdom

${ }^{31}$ University College London, London WC1E 6BT, United Kingdom

${ }^{32}$ Centro de Investigaciones Energeticas Medioambientales y Tecnologicas, E-28040 Madrid, Spain

${ }^{33}$ Massachusetts Institute of Technology, Cambridge, Massachusetts 02139, USA

${ }^{34}$ Institute of Particle Physics: McGill University, Montréal, Québec, H3A 2T8, Canada;

Simon Fraser University, Burnaby, British Columbia, V5A 1S6, Canada;

University of Toronto, Toronto, Ontario, M5S 1A7, Canada;

and TRIUMF, Vancouver, British Columbia, V6T 2A3, Canada

${ }^{35}$ University of Michigan, Ann Arbor, Michigan 48109, USA

${ }^{36}$ Michigan State University, East Lansing, Michigan 48824, USA

${ }^{37}$ Institution for Theoretical and Experimental Physics, ITEP, Moscow 117259, Russia

${ }^{38}$ University of New Mexico, Albuquerque, New Mexico 87131, USA

${ }^{39}$ Northwestern University, Evanston, Illinois 60208, USA

${ }^{40}$ The Ohio State University, Columbus, Ohio 43210, USA

${ }^{41}$ Okayama University, Okayama 700-8530, Japan

${ }^{42}$ Osaka City University, Osaka 588, Japan

${ }^{43}$ University of Oxford, Oxford OX1 3RH, United Kingdom

${ }^{44 \mathrm{a}}$ Istituto Nazionale di Fisica Nucleare, Sezione di Padova-Trento, I-35131 Padova, Italy

${ }^{44 \mathrm{~b}}$ University of Padova, I-35131 Padova, Italy

${ }^{45}$ LPNHE, Universite Pierre et Marie Curie/IN2P3-CNRS, UMR7585, Paris, F-75252 France

${ }^{46}$ University of Pennsylvania, Philadelphia, Pennsylvania 19104, USA

${ }^{47 a}$ Istituto Nazionale di Fisica Nucleare Pisa, I-56127 Pisa, Italy

${ }^{47 \mathrm{~b}}$ University of Pisa, I-56127 Pisa, Italy

${ }^{47 \mathrm{c}}$ University of Siena, I-56127 Pisa, Italy

${ }^{47 \mathrm{~d}}$ Scuola Normale Superiore, I-56127 Pisa, Italy

${ }^{48}$ University of Pittsburgh, Pittsburgh, Pennsylvania 15260, USA

${ }^{49}$ Purdue University, West Lafayette, Indiana 47907, USA

${ }^{50}$ University of Rochester, Rochester, New York 14627, USA

${ }^{51}$ The Rockefeller University, New York, New York 10021, USA

${ }^{52 a}$ Istituto Nazionale di Fisica Nucleare, Sezione di Roma 1, I-00185 Roma, Italy

${ }^{52 \mathrm{~b}}$ Sapienza Università di Roma, I-00185 Roma, Italy

${ }^{53}$ Rutgers University, Piscataway, New Jersey 08855, USA

${ }^{54}$ Texas A\&M University, College Station, Texas 77843, USA

${ }^{55 a}$ Istituto Nazionale di Fisica Nucleare Trieste/Udine, I-34100 Trieste, Italy

${ }^{55 \mathrm{~b}}$ University of Trieste/Udine, I-33100 Udine, Italy

${ }^{56}$ University of Tsukuba, Tsukuba, Ibaraki 305, Japan

${ }^{57}$ Tufts University, Medford, Massachusetts 02155, USA

${ }^{58}$ Waseda University, Tokyo 169, Japan

${ }^{59}$ Wayne State University, Detroit, Michigan 48201, USA

${ }^{60}$ University of Wisconsin, Madison, Wisconsin 53706, USA

${ }^{61}$ Yale University, New Haven, Connecticut 06520, USA

${ }^{62}$ Center for High Energy Physics: Kyungpook National University, Daegu 702-701, Korea; Seoul National University, Seoul 151-742, Korea; Sungkyunkwan University, Suwon 440-746, Korea;

Korea Institute of Science and Technology Information, Daejeon, 305-806, Korea;

Chonnam National University, Gwangju, 500-757, Korea

(Received 28 May 2009; published 27 August 2009) 
We present the first observation in hadronic collisions of the electroweak production of vector boson pairs $(V V, V=W, Z)$ where one boson decays to a dijet final state. The data correspond to $3.5 \mathrm{fb}^{-1}$ of integrated luminosity of $p \bar{p}$ collisions at $\sqrt{s}=1.96 \mathrm{TeV}$ collected by the CDF II detector at the Fermilab Tevatron. We observe $1516 \pm 239$ (stat) \pm 144 (syst) diboson candidate events and measure a cross section $\sigma(p \bar{p} \rightarrow V V+X)$ of $18.0 \pm 2.8$ (stat) \pm 2.4 (syst) \pm 1.1 (lumi) $\mathrm{pb}$, in agreement with the expectations of the standard model.

DOI: 10.1103/PhysRevLett.103.091803

PACS numbers: 14.80.Bn, 12.15.-y, 14.70.-e

The production of heavy gauge boson pairs ( $W W, W Z$, or $Z Z$ ) in $p \bar{p}$ collisions has been observed in the fully leptonic final states at the Fermilab Tevatron collider [1,2]. Diboson production has not yet been conclusively observed in $p \bar{p}$ collisions in decay channels involving hadrons [3]; however, evidence for diboson decays into an $l \bar{\nu} q \bar{q}^{(\prime)}$ final state $(l=e, \mu, \tau ; q=u, d, s, c, b)$ has been recently presented by the D0 collaboration [4].

Measurements of diboson production cross sections provide tests of the self-interactions of the gauge bosons. Deviations from the standard model (SM) prediction for the production rates could indicate new physics [5,6]. Furthermore, given that diboson production is topologically similar to associated Higgs boson production, $p \bar{p} \rightarrow$ $V H+X(V=W, Z)$, the analysis techniques described in this Letter are important for Higgs boson searches.

Here, we present the first observation at a hadron collider of diboson production with one boson decaying into leptons and the other into hadrons. The analysis is performed on a sample of events with large transverse momentum imbalance $\left(\mathscr{E}_{T}\right)$ and two jets whose invariant mass can be reconstructed. This signature is sensitive not only to $l \bar{\nu} q \bar{q}^{(\prime)}$, but also to $\nu \bar{\nu} q \bar{q}^{(\prime)}$ decays because we do not explicitly require presence of identified charged leptons. The limited dijet mass resolution results in a significant overlap of the $W \rightarrow q \bar{q}^{\prime}$ and the $Z \rightarrow q \bar{q}$ dijet mass peaks, and therefore the combination of the three diboson signals, $W W, W Z$, and $Z Z$, is considered.

We analyze a dataset of $p \bar{p}$ collisions corresponding to an integrated luminosity of $3.5 \mathrm{fb}^{-1}$ collected with the CDF II detector at the center-of-mass energy of $1.96 \mathrm{TeV}$. The CDF II detector is described in detail elsewhere [7]. The detector is cylindrically symmetric around the proton beam axis which is oriented in the positive $z$ direction. The polar angle, $\theta$, is measured from the origin of the coordinate system at the center of the detector with respect to the $z$ axis. The pseudorapidity, transverse energy, and transverse momentum are defined as $\eta=$ - ln $\tan (\theta / 2), E_{T}=E \sin (\theta)$, and $p_{T}=p \sin (\theta)$, respectively. The central and plug calorimeters, which, respectively, cover the pseudorapidity regions of $|\eta|<1.1$ and $1.1<|\eta|<3.6$, surround the tracking system with a projective tower geometry. The missing $E_{T}$ is defined by $\mathbb{E}_{T}=$ $\left|\vec{E}_{T}\right|, \vec{E}_{T}=-\sum_{i} E_{T}^{i} \hat{\mathbf{n}}_{\mathbf{i}}$, where $\hat{\mathbf{n}}_{\mathbf{i}}$ is a unit vector perpendicular to the beam axis and pointing at the $i$ th calorimeter tower. The sum $E_{T}$ is defined by $\sum E_{T}=\sum_{i} E_{T}^{i}$. Both sums are over all calorimeter towers with $E_{T}>100 \mathrm{MeV}$.

The diboson signal $(W W, W Z$, and $Z Z)$ is simulated using the PYTHIA V6.2 Monte Carlo generator [8]. The most significant backgrounds to the diboson signal are $W(l \bar{\nu})+$ jets, $Z(\nu \bar{\nu})+$ jets, and QCD multijet production (in the following referred to as multijet background or MJB). Other less significant backgrounds include $Z(l \bar{l})+$ jets, $t \bar{t}$, and single $t$-quark production. The $W+$ jets backgrounds are simulated using the fixed-order matrix element generator ALPGEN V2.1 [9] which is interfaced with PYTHIA V6.3 to simulate parton showering and fragmentation, the underlying event, and additional $p \bar{p}$ interactions in the same bunch crossing. The $Z+$ jets and $t$-quark production processes are simulated with PYTHIA V6.2. The detector response in all Monte Carlo samples is modeled by a GEANT-based CDF II detector simulation [10]. The MJB does not typically result in signatures of large intrinsic $\mathscr{E}_{T}$. However, when jet energy is not measured accurately, an event may be reconstructed with large $\mathscr{E}_{T}$ and pass the analysis selection criteria. Because of the large multijet production rate, this can still be a significant background in a $\not_{T}+$ jets based analysis. The MJB is determined from the data. All other background predictions are normalized using next-to-leading-order (NLO) calculations for SM cross sections.

The selection of signal proceeds as follows: first events are selected by a set of hardware triggers, then a series of analysis cuts reduces the backgrounds, and finally the signal is extracted using a minimization of a fitting procedure. The hardware triggers have benefited significantly from the calorimeter trigger upgrade completed in 2007 [11]. The majority (94\%) of events satisfy the inclusive $\mathbb{E}_{T}$ trigger, which requires $\mathbb{E}_{T}>45 \mathrm{GeV}$. Jets are reconstructed in the calorimeter using the JETCLU cone algorithm [12] with a cone radius of 0.4 in $(\eta, \phi)$ space. We select events that have $\mathbb{E}_{T}>60 \mathrm{GeV}$ and exactly two reconstructed jets with $E_{T}>25 \mathrm{GeV}$ and $|\eta|<2.0$. This ensures a trigger efficiency of $96 \% \pm 2 \%$ on signal. We search for diboson production in the dijet mass range $40<$ $M_{j j}<160 \mathrm{GeV} / c^{2}$. The lower edge of the mass range is chosen to ensure that events are on the trigger efficiency plateau with respect to dijet mass. In addition to the requirements discussed above, the electromagnetic fraction of the total energy for each of the two jets is required to 
be less than $90 \%$ to ensure that electrons and photons are not counted as jets.

In order to suppress the $\mathrm{MJB}$, we use a $\mathbb{E}_{T}$ resolution model to distinguish true $\mathscr{E}_{T}$ originating from undetected neutrinos from fake $\mathscr{E}_{T}$ due to jets that are not measured accurately. The $\not{E}_{T}$ significance is a dimensionless quantity based on the energy resolution of the jets, on soft unclustered particles, and on the event topology. The $\mathbb{E}_{T}$ significance is typically low when $\mathscr{E}_{T}$ arises from mismeasurement. In addition to having a small significance, the $\overrightarrow{\boldsymbol{E}}_{T}$ will often be aligned with a jet. We select events with $\mathscr{E}_{T}$ significance larger than 4 and azimuthal angle between $\vec{E}_{T}$ and the nearest jet $\left(\Delta \phi_{\mathbb{E}_{T}}^{\text {jet }}\right)$ greater than 0.4 radians.

Finally, we apply several requirements that suppress contamination due to cosmic-ray, beam-related, and other noncollision backgrounds. Events are required to have at least one reconstructed vertex formed by charged particle tracks. The transverse energies of all calorimeter towers are calculated with respect to the $z$ position of the primary vertex with the largest $\sum p_{T}$ of associated tracks. The electromagnetic fraction of the total event energy has to be larger than $30 \%$ in order to reduce beam-related backgrounds. The arrival time of both leading jets as measured by the electromagnetic shower timing system [13] has to be consistent with the $p \bar{p}$ collision time. The remaining noncollision background has a smooth $M_{j j}$ distribution and accounts for less than $0.2 \%$ of the final number of selected events. After all cuts were applied, we find 44910 events in the final sample.

The shape and normalization of the MJB are determined from the data. A vector, $\vec{p}_{T}$, analogous to the calorimeterbased $\vec{E}_{T}$, is constructed from the vector sum of the transverse momenta of particles measured in the tracking system, and is largely uncorrelated to $\vec{E}_{T}$ for events where jets are not reconstructed accurately. In the absence of $\mathscr{E}_{T}$ arising from mismeasurement in the calorimeter, the $\vec{E}_{T}$ and $\vec{p}_{T}$ will be aligned in most events. The MJB is expected to be the dominant background component at larger values of $\Delta \phi\left(\vec{E}_{T}, \overrightarrow{\not p}_{T}\right)$. The dijet mass shape and normalization for the remaining MJB contribution in the sample is found by selecting events with $\Delta \phi\left(\overrightarrow{\mathscr{E}}_{T}, \vec{p}_{T}\right)>1.0$ and subtracting out the non-MJB backgrounds. The normalization is scaled up to account for the MJB contamination in the region $\Delta \phi\left(\vec{E}_{T}, \vec{p}_{T}\right)<1.0$. The shape of the MJB is fit to an exponential in $M_{j j}$ to derive a dijet mass template. The MJB shapes of $M_{j j}$ and $\Delta \phi\left(\vec{E}_{T}, \vec{p}_{T}\right)$ distributions are verified with a large statistics MC sample.

The signal extraction is performed using a minimization of the unbinned extended negative log likelihood with the ROOFIT program [14]. Three $M_{j j}$ template distributions are used in the fit: the first is $V+$ jets and $t$-quark production [in the following referred to as "electroweak" (EWK) backgrounds] and is taken from Monte Carlo simulation; the second is the MJB template, where the slope and normalization are Gaussian constrained to their previously measured values; the third template describes the signal. The signal shape is comprised of the $W W, W Z$, and $Z Z$ distributions. This template is obtained from a Gaussian + polynomial fit to the signal Monte Carlo simulation where the mean and the width of the Gaussian distribution are linearly dependent on the jet energy scale (JES).

To assess the effect of systematic uncertainties on the measurement, we address separately two classes of sources: those that affect the signal extraction procedure and those that affect the signal acceptance in the cross section calculation. The signal extraction systematic uncertainties come from uncertainties in signal and background shapes. The shape uncertainties take into account the effect of jet energy resolution (JER), JES, MJB shape, and the shape of the EWK background. The jet energy scale and the shape and the normalization of MJB are treated as nuisance parameters in the fit and Gaussian constrained to their independently measured values. These uncertainties are therefore accounted for in the statistical uncertainty of the extraction.

The shape uncertainty for the EWK background is determined by using $\gamma+$ jets data [15] as an alternative background model in the $M_{j j}$ fit. All major non-MJB backgrounds include a gauge boson accompanied by jets. There are similarities between the $\gamma+$ jets and $V+$ jets production; however, due largely to the mass difference between the $\gamma$ and the $W / Z$, the kinematics is not identical. To take this into account, the $\gamma+$ jets data are weighted by the ratio of the dijet mass distributions of the EWK background MC samples to $\gamma+$ jets PYTHIA MC sample. We use these adjusted $\gamma+$ jets data to determine a systematic uncertainty on the EWK $M_{j j}$ template. Selection cuts applied to the $\gamma+$ jets events are not identical to those applied to the $\mathbb{E}_{T}+$ jets sample. For example, the $Z$ decay into neutrinos will register as $\mathbb{E}_{T}$ in the detector, while the photon $E_{T}$ will be measured in the calorimeter. For this reason, we cut on the vector sum of the photon $E_{T}$ and any $\mathscr{E}_{T}$ present in $\gamma+$ jets events at $60 \mathrm{GeV}$, treating this sum as analogous to $\mathbb{E}_{T}$ in $V+$ jets events. A further consideration in the construction of the $\gamma+$ jets template is the effect of $\gamma+V$ events, as these events will cause a peak in the $\gamma+$ jets dijet mass distribution. We subtract this contribution using the $\gamma+V$ PYTHIA sample. Finally, we perform two signal extraction fits using the default EWK and $\gamma+$ jets templates, respectively. The uncertainty due to the shape of the EWK background is then estimated as the difference in the results obtained from these two fits. The described method accounts for a combined effect of JES, JER, and modeling of jets in MC simulations on the EWK $M_{j j}$ template.

The uncertainty associated with the JES is the dominant source of systematic uncertainty on the acceptance and, 
TABLE I. The systematic uncertainties and their effect on the number of extracted signal events, the acceptance, and the cross section. All systematics are added in quadrature.

\begin{tabular}{llc}
\hline \hline & \multicolumn{1}{c}{ Systematic } & $\%$ uncert. \\
\hline \multirow{2}{*}{ Extraction } & EWK shape & 7.7 \\
& Resolution & 5.6 \\
& Total extraction & 9.5 \\
& JES & 8.0 \\
Acceptance & JER & 0.7 \\
& E $_{T}$ resolution model & 1.0 \\
& Trigger inefficiency & 2.2 \\
& ISR/FSR & 2.5 \\
& PDF & 2.0 \\
& Total acceptance & 9.0 \\
& Total systematics & 13.1 \\
& Luminosity & 5.9 \\
& Total & 14.4 \\
\hline \hline
\end{tabular}

therefore, the cross section. Other less significant sources of systematic uncertainty that affect the measured cross section are jet energy resolution, initial and final state radiation (ISR/FSR), and parton distribution functions (PDF). A summary of all sources of systematic uncertainty is presented in Table I.

The measured yields for signal and backgrounds are given in Table II. Based on the MC simulation, the acceptances for the $W W, W Z$, and $Z Z$ production is $2.5 \%, 2.6 \%$, and $2.9 \%$, respectively. In the calculation of the combined diboson cross section, we assume that each signal process contributes proportionally to its predicted SM cross section: $11.7 \mathrm{pb}$ for $W W, 3.6 \mathrm{pb}$ for $W Z$, and $1.5 \mathrm{pb}$ for $Z Z$. The number of signal events we extract [1516 \pm 239 (stat) \pm 144 (syst)] corresponds to a cross section of $18.0 \pm 2.8$ (stat) \pm 2.4 (syst) \pm 1.1 (lumi) $\mathrm{pb}$, in agreement with the SM prediction of $16.8 \pm 0.5 \mathrm{pb}$ obtained using the MCFM V5.4 program [16] with CTEQ6.1M PDFs [17].

Figure 1 shows a comparison between the observed $\Delta \phi_{\mathbb{L}_{T}}^{\text {jet }}$ distribution and the MJB and EWK (signal +

TABLE II. Value of parameters in the model used to fit the $M_{j j}$ distribution. $1516 \pm 239$ (stat) signal events are extracted from the 44910 data events which pass our selection cuts. The jet energy scale is also extracted from the fit and agrees well with the default value (1.0) as measured from calibrations.

\begin{tabular}{lr}
\hline \hline \multicolumn{1}{c}{ Parameter } & Fitted value \\
\hline Jet energy scale, JES & $0.985 \pm 0.019$ \\
Yield of EWK background events & $36,140 \pm 1230$ \\
Yield of MJB events & $7249 \pm 1130$ \\
Yield of diboson candidates & $1516 \pm 239$ \\
\hline \hline
\end{tabular}

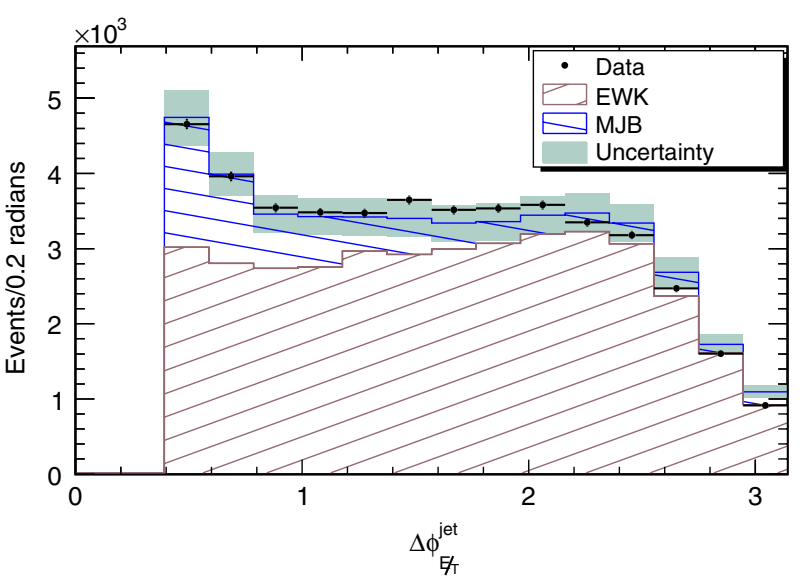

FIG. 1 (color online). Data compared with the sum of the predicted EWK and MJB backgrounds for the $\Delta \phi_{\mathscr{Z}_{T}}^{\text {jet }}$ variable. The band represents the total systematic uncertainty on the background. The measured signal is included here in the EWK contribution.

background) components. This distribution provides a strong consistency check on our MJB model. Figure 2 shows the fit result and a comparison between the expected signal and data after background subtraction. We bin the data as in Fig. 2 and obtain a $\chi^{2}$ of 9.4 for 9 degrees of freedom corresponding to a $p$ value of $40 \%$.

In summary, we use the $\not{E}_{T}+$ jets final state to measure the $W W+W Z+Z Z$ cross section in $p \bar{p}$ collisions at

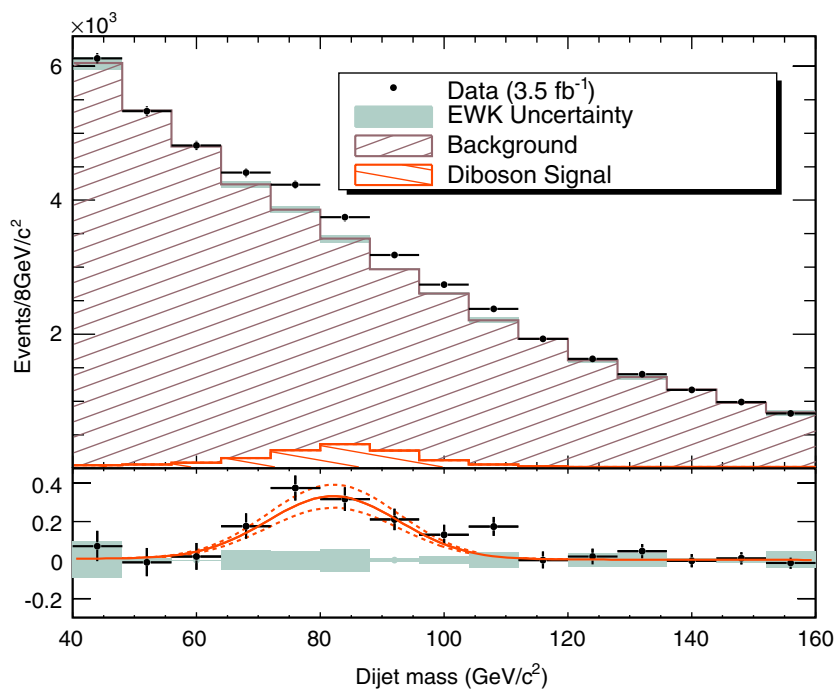

FIG. 2 (color online). Top: Comparison between data and fitted background only. The measured signal is shown unstacked. The band represents the systematic uncertainty due to the shape of EWK background as described in the text. Bottom: Comparison of the diboson signal (solid line) with the backgroundsubtracted data (points). The dashed lines represent the $\pm 1 \sigma$ statistical variations on the extracted signal. The gray band represents the systematic uncertainty due to the EWK shape. 
$\sqrt{s}=1.96 \mathrm{TeV} \quad$ to $\quad$ be $18.0 \pm 2.8$ (stat) $\pm 2.4($ syst $) \pm$ 1.1(lumi) pb. This is consistent with the SM prediction of $16.8 \pm 0.5 \mathrm{pb}$. To assess the strength of the observed signal, the effects of parameter variations due to all relevant sources of uncertainty are studied by comparing the likelihood of the background-only fit with the full fit result, and converting the difference into significance numbers. We thus measure that the signal corresponds to a significance of at least 5.3 standard deviations from the background-only hypothesis. This is the first time the vector boson pair production has been observed in a hadronic final state at the Tevatron collider.

We thank the Fermilab staff and the technical staffs of the participating institutions for their vital contributions. This work was supported by the U.S. Department of Energy and National Science Foundation; the Italian Istituto Nazionale di Fisica Nucleare; the Ministry of Education, Culture, Sports, Science and Technology of Japan; the Natural Sciences and Engineering Research Council of Canada; the National Science Council of the Republic of China; the Swiss National Science Foundation; the A.P. Sloan Foundation; the Bundesministerium für Bildung und Forschung, Germany; the Korean Science and Engineering Foundation and the Korean Research Foundation; the Science and Technology Facilities Council and the Royal Society, UK; the Institut National de Physique Nucleaire et Physique des Particules/CNRS; the Russian Foundation for Basic Research; the Ministerio de Ciencia e Innovación, and Programa ConsoliderIngenio 2010, Spain; the Slovak R\&D Agency; and the Academy of Finland.

\footnotetext{
${ }^{\mathrm{a}}$ Deceased.

${ }^{\mathrm{b}}$ Visitors from University of Massachusetts Amherst, Amherst, MA 01003, USA.

${ }^{\mathrm{c}}$ Visitors from Universiteit Antwerpen, B-2610 Antwerp, Belgium.

${ }^{\mathrm{d}}$ Visitors from University of Bristol, Bristol BS8 1TL, United Kingdom.

${ }^{\mathrm{e}}$ Visitors from Chinese Academy of Sciences, Beijing 100864, China.

${ }^{\mathrm{f}}$ Visitors from Istituto Nazionale di Fisica Nucleare, Sezione di Cagliari, 09042 Monserrato (Cagliari), Italy.

${ }^{\mathrm{g}}$ Visitors from University of California Irvine, Irvine, CA 9269., USA.

${ }^{\mathrm{h}}$ Visitors from University of California Santa Cruz, Santa Cruz, CA 95064, USA.

${ }^{i}$ Visitors from Cornell University, Ithaca, NY 14853, USA. ${ }^{j}$ Visitors from University of Cyprus, Nicosia CY-1678, Cyprus.

${ }^{k}$ Visitors from University College Dublin, Dublin 4, Ireland.

${ }^{1}$ Visitors from University of Edinburgh, Edinburgh EH9 3JZ, United Kingdom.
}

${ }^{\mathrm{m}}$ Visitors from University of Fukui, Fukui City, Fukui Prefecture, Japan 910-0017.

${ }^{n}$ Visitors from Kinki University, Higashi-Osaka City, Japan $577-8502$

${ }^{\circ}$ Visitors from Universidad Iberoamericana, Mexico D.F., Mexico.

${ }^{\mathrm{P}}$ Visitors from University of Iowa, Iowa City, IA 52242, USA.

${ }^{\mathrm{q}}$ Visitors from Queen Mary, University of London, London, E1 4NS, England.

${ }^{r}$ Visitors from University of Manchester, Manchester M13 9PL, England.

${ }^{\mathrm{s}}$ Visitors from Nagasaki Institute of Applied Science, Nagasaki, Japan.

${ }^{t}$ Visitors from University of Notre Dame, Notre Dame, IN 46556, USA.

uisitors from University de Oviedo, E-33007 Oviedo, Spain.

${ }^{\mathrm{v}}$ Visitors from Texas Tech University, Lubbock, TX 79609 , USA.

${ }^{\text {w}}$ Visitors from IFIC (CSIC-Universitat de Valencia), 46071 Valencia, Spain.

${ }^{\mathrm{x}}$ Visitors from University of Virginia, Charlottesville, VA 22904, USA.

${ }^{\mathrm{y}}$ Visitors from Bergische Universität Wuppertal, 42097 Wuppertal, Germany.

${ }^{\mathrm{z} V i s i t o r s ~ f r o m ~ O n ~ l e a v e ~ f r o m ~ J . ~ S t e f a n ~ I n s t i t u t e, ~ L j u b l j a n a, ~}$ Slovenia.

[1] D. Acosta et al. (CDF collaboration), Phys. Rev. Lett. 94, 211801 (2005); A. Abulencia et al., ibid. 98, 161801 (2007); T. Aaltonen et al., ibid. 100, 201801 (2008).

[2] V. M. Abazov et al. (D0 collaboration), Phys. Rev. Lett. 94, 151801 (2005); Phys. Rev. D 76, 111104(R) (2007).

[3] T. Aaltonen et al. (CDF collaboration), Phys. Rev. D 76, 111103 (2007).

[4] V. M. Abazov et al. (D0 collaboration), Phys. Rev. Lett. 102, 161801 (2009).

[5] K. Hagiwara et al., Nucl. Phys. B282, 253 (1987).

[6] M. Kober, B. Koch, and M. Bleicher, Phys. Rev. D 76, 125001 (2007).

[7] A. Abulencia et al. (CDF collaboration), J. Phys. G 34, 2457 (2007).

[8] T. Sjostrand et al., Comput. Phys. Commun. 135, 238 (2001).

[9] M. L. Mangano et al., J. High Energy Phys. 07 (2003) 001.

[10] E. Gerchtein and M. Paulini, eConf C0303241, TUMT005 (2003).

[11] A. Bhatti et al., IEEE Trans. Nucl. Sci. 56, 1685 (2009).

[12] A. F. Abe et al. (CDF collaboration), Phys. Rev. D 45, 1448 (1992).

[13] M. Goncharov et al., Nucl. Instrum. Methods Phys. Res., Sect. A 565, 543 (2006).

[14] W. Verkerke and D. Kirby, arXiv:physics/0306116.

[15] F. Abe et al. (CDF collaboration), Phys. Rev. Lett. 81, 1791 (1998) contains standard photon identification criteria.

[16] J. M. Campbell and R. K. Ellis, Phys. Rev. D 60, 113006 (1999).

[17] J. Pumplin et al., J. High Energy Phys. 07 (2002) 012. 